Check for updates

Cite this: Chem. Sci., 2020, 11, 508

- All publication charges for this article have been paid for by the Royal Society of Chemistry

Received 2nd August 2019

Accepted 18th November 2019

DOI: $10.1039 / c 9 s c 03854 j$

rsc.li/chemical-science

\title{
IMPRESSION - prediction of NMR parameters for 3-dimensional chemical structures using machine learning with near quantum chemical accuracy $\dagger$
}

\author{
Will Gerrard, (D) a Lars A. Bratholm, (D) a Martin J. Packer, ${ }^{b}$ Adrian J. Mulholland, (D) ${ }^{a}$ \\ David R. Glowacki (D)*a and Craig P. Butts ${ }^{*}$ *a
}

\begin{abstract}
The IMPRESSION (Intelligent Machine PREdiction of Shift and Scalar information Of Nuclei) machine learning system provides an efficient and accurate method for the prediction of NMR parameters from 3-dimensional molecular structures. Here we demonstrate that machine learning predictions of NMR parameters, trained on quantum chemical computed values, can be as accurate as, but computationally much more efficient (tens of milliseconds per molecular structure) than, quantum chemical calculations (hours/days per molecular structure) starting from the same 3-dimensional structure. Training the machine learning system on quantum chemical predictions, rather than experimental data, circumvents the need for the existence of large, structurally diverse, error-free experimental databases and makes IMPRESSION applicable to solving 3-dimensional problems such as molecular conformation and stereoisomerism.
\end{abstract}

\section{Introduction}

NMR spectroscopy remains the pre-eminent analytical technique for elucidating molecular structure in solution, with the prediction and interpretation of ${ }^{1} \mathrm{H}$ and ${ }^{13} \mathrm{C}$ chemical shifts and scalar coupling constants playing a key role. The prediction of these parameters, especially in studies of 3 -dimensional molecular structure, are increasingly moving towards quantitative comparison between computed values for proposed chemical structures and experiment. In such comparisons, the use of fast and accurate NMR prediction methods is crucial.

Fast empirical predictions of chemical shifts for 2-dimensional chemical structures have been used for decades, with the additivity rules exemplified by Pretsch ${ }^{1}$ and HOSE-code ${ }^{2}$ variants forming the basis of many analyses. However their applicability is limited by being based on 2-dimensional structures and cannot readily deal with 3-dimensional conformational or stereochemical analysis. Some modifications to treating 3dimensional structures have been made by e.g. flat-butstereochemically-aware HOSE codes $^{3}$ or single conformer models of experimental system $\mathrm{s}^{4-6}$ but the improvements in 3dimensional accuracy are limited as conformation and flexibility must necessarily be accounted for completely to achieve maximum accuracy. Multiple-bond ${ }^{1} \mathrm{H}^{-}{ }^{1} \mathrm{H}$ coupling constants

${ }^{a}$ University of Bristol, Bristol, UK. E-mail: craig.butts@bristol.ac.uk; glowacki@bristol. ac.uk

${ }^{b}$ Chemistry, R\&D Oncology, AstraZeneca, Cambridge CB4 OQA, UK

$\dagger$ Electronic supplementary information (ESI) available. See DOI: $10.1039 / \mathrm{c} 9 \mathrm{sc} 03854 \mathrm{j}$ are more directly linked to 3-dimensional structure, however generically applicable Karplus-style empirical relationships, such as the widely used equation reported by Haasnoot et al., ${ }^{7}$ suffer from lower accuracy when confronted with complex chemical functionality while equations designed for specific sub-structures, e.g. carbohydrates, ${ }^{8}$ are not applicable to the whole of chemical space. Finally, many NMR parameters, for example 1-bond ${ }^{1} \mathrm{H}_{-}{ }^{13} \mathrm{C}$ scalar coupling constants, ${ }^{1} J_{\mathrm{CH}}$, which are sensitive to both chemical connectivity and 3-dimensional structure are rarely used in isotropic studies precisely because there are no general fast predictive methods for ${ }^{1} J_{\mathrm{CH}}$.

For all of these reasons, the accurate prediction of NMR parameters in modern 3-dimensional structure determinations relies increasingly on the use of quantum chemical calculations, typically based on Density Functional Theory (DFT) ${ }^{9-12}$ Optimal DFT methods can be accurate to within $1-2 \%$, e.g. ${ }^{1} J_{\mathrm{CH}}$ predicted with $<4 \mathrm{~Hz}$ accuracy to experiment ${ }^{13-15}$ (on values that range from roughly $100-250 \mathrm{~Hz}$ ) and $<0.2 /<2 \mathrm{ppm}^{16,17}$ (on ranges of $\sim 10 / \sim 200 \mathrm{ppm}$ ) for ${ }^{1} \mathrm{H}$ and ${ }^{13} \mathrm{C}$ chemical shifts respectively. The substantial downside of DFT is the significant computation time required when using methods that can provide sufficient accuracy in NMR predictions. Accurate DFT-based predictions of chemical shift and scalar couplings typically take hours to days of CPU time for a single rigid molecule of even relatively low ( 500) molecular mass. The largest proportion of this CPU time is occupied by the NMR computations, especially when computing scalar coupling constants. Naturally, in cases where multiple conformers or isomers must be considered (and thus predictions for multiple structures are required) this becomes days to months of computation for a single study. 
Machine learning methods offer a solution to the timedemands of DFT NMR predictions, achieving them in seconds rather than hours or days. Such machines, trained on experimental data, for ${ }^{1} \mathrm{H}$ and ${ }^{13} \mathrm{C}$ chemical shifts based on 2dimensional structures are well-established. ${ }^{18-21}$ These systems are trained on hundreds of thousands of validated experimental chemical shifts arising from tens of thousands of chemical structures. Training such machines for prediction of scalar couplings is more challenging because accurate and validated experimental databases do not exist on this scale (e.g. ${ }^{1} J_{\mathrm{CH}}$ values) and they can be critically dependent on 3-dimensional structure (e.g. ${ }^{3} J_{\mathrm{HH} / \mathrm{CH}}$ values). On the other hand, a machine could be trained using large datasets of DFT-computed NMR parameters, such as chemical shifts and scalar couplings, derived from 3-dimensional structures. Such large DFT-derived datasets can be generated systematically with minimal effort and are not limited to offering accuracy only for structures that are similar to previously experimentally determined molecules. With a large enough training database, such a machine would be expected to approach the accuracy of DFT calculation of NMR parameters for 3-dimensional structure analysis, but with several orders of magnitude reduction in time for the NMR predictions. This approach was recently reported for solid-state chemical shift predictions by Paruzzo et al. (SHIFTML, ${ }^{22}$ ) where the computational demand of DFT calculations on extended lattices are high and comparable to those needed for multiconformer calculations on solution-state systems.

In this paper we describe the development of our first generation of solution-state NMR prediction machines IMPRESSION (Intelligent Machine PREdiction of Shift and Scalar information Of Nuclei), trained on DFT-predicted values rather than relying on scarce or error-prone experimental data. We have chosen to demonstrate the versatility of machine learning of NMR parameters using both ${ }^{1} \mathrm{H}$ and ${ }^{13} \mathrm{C}$ chemical shifts and ${ }^{1} J_{\mathrm{CH}}$ couplings. We include scalar couplings in addition to chemical shift, as the former are less amenable to machine learning based on experimental data, and ${ }^{1} J_{\mathrm{CH}}$ precisely because it has been demonstrated to be valuable for elucidating both 2-dimensional connectivity and 3-dimensional structure $^{5,23}$ but requires DFT to predict/interpret for most cases. Providing a fast and accurate predictive tool for ${ }^{1} J_{\mathrm{CH}}$ will be especially valuable and could encourage wider acceptance of this and other accessible NMR parameters in structure determinations. We demonstrate that IMPRESSION can predict all these NMR parameters for organic molecules, including 3dimensional discrimination, with up to DFT accuracy but several orders of magnitude faster and can be applied to experimental data with comparable outcomes to DFT.

\section{Results and discussion}

\subsection{Dataset production and framework}

In order to train and test IMPRESSION, we developed a dataset of NMR parameters $\left(\delta^{1} \mathrm{H}, \delta^{13} \mathrm{C},{ }^{1} J_{\mathrm{CH}}\right)$, computed using DFT in the Gaussian09 software package. ${ }^{24}$ While more demanding computational methods could be considered, ${ }^{25}$ their computational cost would be extortionate with minimal improvement in outcomes for the training and testing datasets described. Instead we found that using mPW1PW91/6-311g(d,p) for optimisation and $\omega \mathrm{b} 97 \mathrm{xd} / 6-311 \mathrm{~g}(\mathrm{~d}, \mathrm{p})^{26-30}$ for computing the NMR parameters was computationally efficient and sufficiently accurate for comparison to experimental values across a range of NMR parameters. In the geometry optimisations a tight optimisation criteria and ultrafine integral grids were used to minimise molecular orientation affecting geometries and energies (see ref. 31 and references therein for a discussion of this). The NMR parameters were calculated using gauge independent atomic orbitals with uncontracted basis sets to improve descriptions of the core orbitals ${ }^{30}$ and calculation of all components of the scalar couplings (Fermi contact, spin dipole, diamagnetic spin orbit, paramagnetic spin orbit). The calculated magnetic shielding tensors were converted into chemical shifts using the linear scaling method and reference compounds reported by Tantillo et al. ${ }^{\mathbf{1 0 , 3 2}} \mathrm{A}$ training set of 882 structures (17 $222{ }^{1} J_{\mathrm{CH}} ; 18383 \quad \delta^{1} \mathrm{H} ; 17081 \quad \delta^{13} \mathrm{C}$ values/ environments) were selected by an adaptive sampling (active learning) procedure ${ }^{33-35}$ from a superset of 75382 chemical structures comprising only $\mathrm{C}, \mathrm{H}, \mathrm{N}, \mathrm{O}$ and $\mathrm{F}$ atoms in the Cambridge Structural Database ${ }^{36}$ (accessed 7/9/2018). The adaptive sampling procedure trains an initial IMPRESSION machine from 100 chemical structures and then uses this machine to predict the parameters for all remaining structures in the superset to measure their variance in a 5 -fold cross validation (i.e. how much a given parameter changes when predicted from 5 separate machines each trained on a different $80 \%$ subset of the current training set). The 100 structures in the superset which show the highest variance are then added to the training dataset and the cycle is iterated (see ESI for further details $\dagger$ ). Adaptive sampling therefore adds the 100 structures at each training iteration which IMPRESSION is the most uncertain about. In doing so, each added structure provides the maximum benefit to the machine and substantially reduces the overall computational cost required to reach a given accuracy. The test set, against which the quality of the IMPRESSION predictions is independently tested, was comprised of a further 410 chemical structures $\left(7788{ }^{1} J_{\mathrm{CH}} ; 7832 \delta^{1} \mathrm{H} ; 7522 \delta^{13} \mathrm{C}\right.$ environments) harvested from the CSD-500 dataset recently reported by Paruzzo et al. $^{22}$

IMPRESSION uses a Kernel Ridge Regression ${ }^{37}$ (KRR) framework to learn the ${ }^{1} J_{\mathrm{CH}}$ scalar couplings and ${ }^{13} \mathrm{C}$ and ${ }^{1} \mathrm{H}$ chemical shifts of molecular structures. KRR was successfully used by Paruzzo et al. to develop SHIFTML. ${ }^{22}$ Neural networks have also been used to predict chemical shifts in small molecules from experimental data, ${ }^{\mathbf{6}, 38,39}$ however we found no clear advantages in using feed forward neural networks in this work as the accuracy was comparable to KRR for the datasets used, with the kernel methods being much faster to train with the given training set size. In order to encode the similarity between chemical environments of each molecular structure we tested three approaches previously described - Coulomb matrices, ${ }^{\mathbf{4 0}}$ aSLATM, ${ }^{41}$ and FCHL $^{42}$ all available from the QML python package. ${ }^{43}$ We refer the reader to Section S1.1 in the ESI $\dagger$ and the respective papers describing each representation for more details. All of these kernel similarity measures compare atomic 

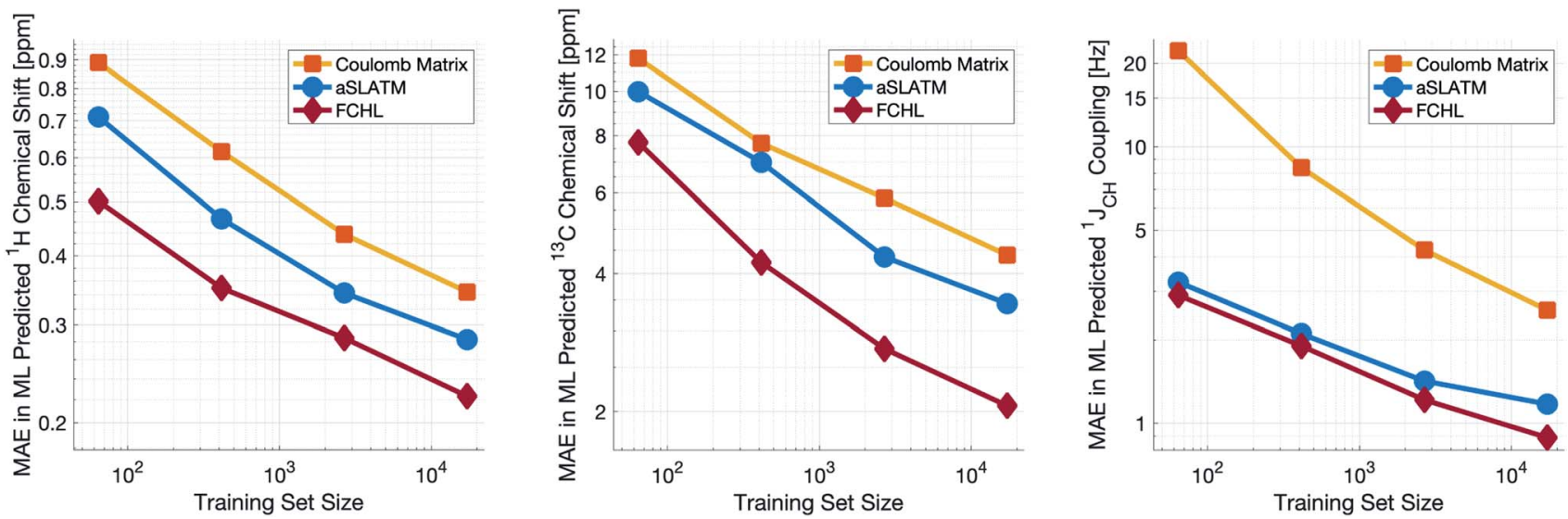

Fig. 1 log-log plot of training set size vs. the mean absolute error between ML predictions and DFT of the test set for $\delta^{1} \mathrm{H}$ (left), $\delta^{13} \mathrm{C}$ (centre) and ${ }^{1} J_{\mathrm{CH}}$ couplings (right). Results are shown for the Coulomb matrix, aSLATM and FCHL kernel similarity measures.

environments, so in the case of ${ }^{1} J_{\mathrm{CH}}$, we used the product of the separately calculated kernel similarities for the ${ }^{1} \mathrm{H}$ and ${ }^{13} \mathrm{C}$ nuclei as this performed better than either atomic environment alone. The KRR procedure is further described in the ESI (Section S1.1†).

Both aSLATM and FCHL were found to outperform Coulomb matrices (Fig. 1), which is expected as Coulomb matrices only include 2-body interactions, while aSLATM and FCHL both include three-body interactions as well. As FCHL provided the best performance for all three parameters and was substantially more computationally efficient than aSLATM, it was used in the final development of the full IMPRESSION machine.

\subsection{Performance relative to DFT}

During training, the machine performance for prediction of all NMR parameters $\left(\delta^{1} \mathrm{H}, \delta^{13} \mathrm{C},{ }^{1} J_{\mathrm{CH}}\right)$ improved steadily with increasing training set size, as illustrated in the learning curves (Fig. 1). This indicates that the accuracy of the machine can be further improved by adding additional training data, however the absolute gains become marginal beyond the dataset size used here with a ten-fold increase in training set size approximately halving the average error between IMPRESSION and DFT. After training on the full set of 882 chemical structures, IMPRESSION predictions achieved mean absolute errors (MAE) of $0.23 \mathrm{ppm} / 2.45 \mathrm{ppm} / 0.87 \mathrm{~Hz}$ for $\delta^{1} \mathrm{H} / \delta^{13} \mathrm{C} /{ }^{1} J_{\mathrm{CH}} /$ predictions and root mean squared error (RMSE) of $0.35 \mathrm{ppm} / 3.88$ $\mathrm{ppm} / 1.39 \mathrm{~Hz}$ against the independent test set (Fig. 2).

Notably however, a very small number of predictions for the test set were much less reliable. For example, $186(\sim 2.3 \%)$ of the $\delta^{1} \mathrm{H}$ values had errors $>1 \mathrm{ppm}$ between IMPRESSION and DFT, with a maximum error (MaxE) of $11.22 \mathrm{ppm}$. Similar outcomes were observed for the other parameters with $187 \delta^{13} \mathrm{C}$ values $(\sim 2.5 \%)$ with errors $>10 \mathrm{ppm}(\operatorname{MaxE}=63.33 \mathrm{ppm})$ and 14 $(\sim 0.2 \%)$ of the 7788 predicted ${ }^{1} J_{\mathrm{CH}}$ values having errors of $>10 \mathrm{~Hz}(\mathrm{MaxE}=24.63 \mathrm{~Hz})$. Diagrams of the structures containing the five most significant outliers for each NMR parameter are shown in Fig. S19-S21 in the ESI. $\dagger$ Examination of the chemical environments of the most significant outliers show that they arise from unusual functional groups such as those containing sp-hybridised atoms, or unusual 3-dimensional environments such as atoms near pi-systems of aromatic rings. These outliers suggest that, as desired, the machine learning
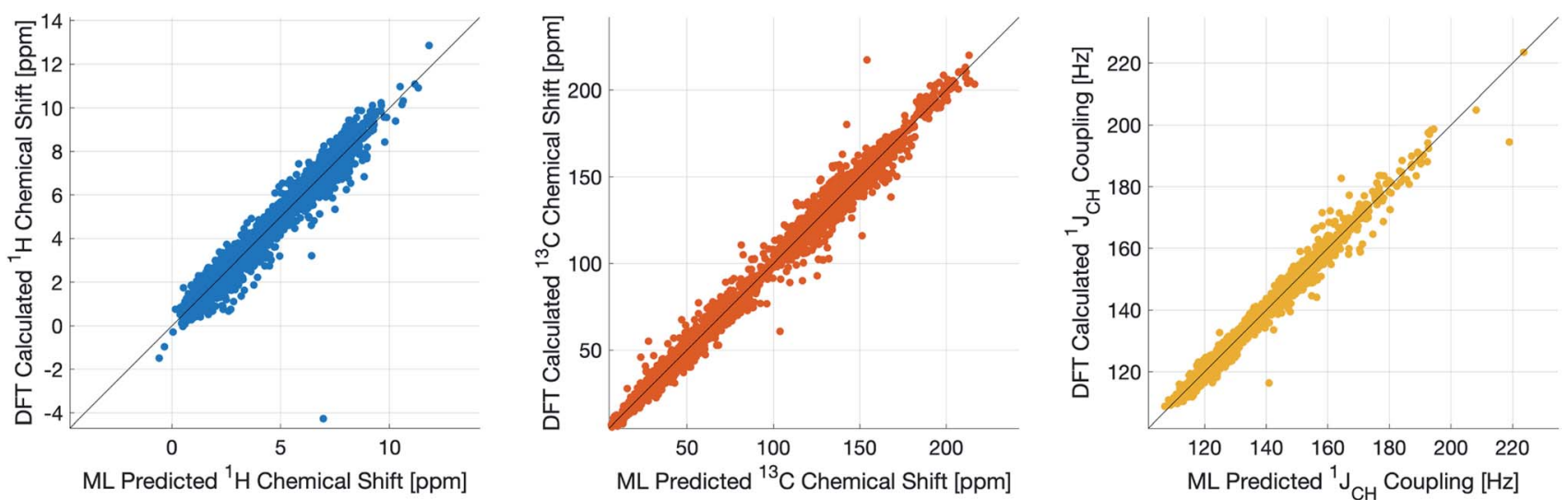

Fig. 2 IMPRESSION machine learning predictions compared to DFT computed NMR parameters for $\delta^{1} \mathrm{H}$ (left), $\delta^{13} \mathrm{C}$ (centre) and ${ }^{1} J_{C H}$ couplings (right) without variance filtering. 

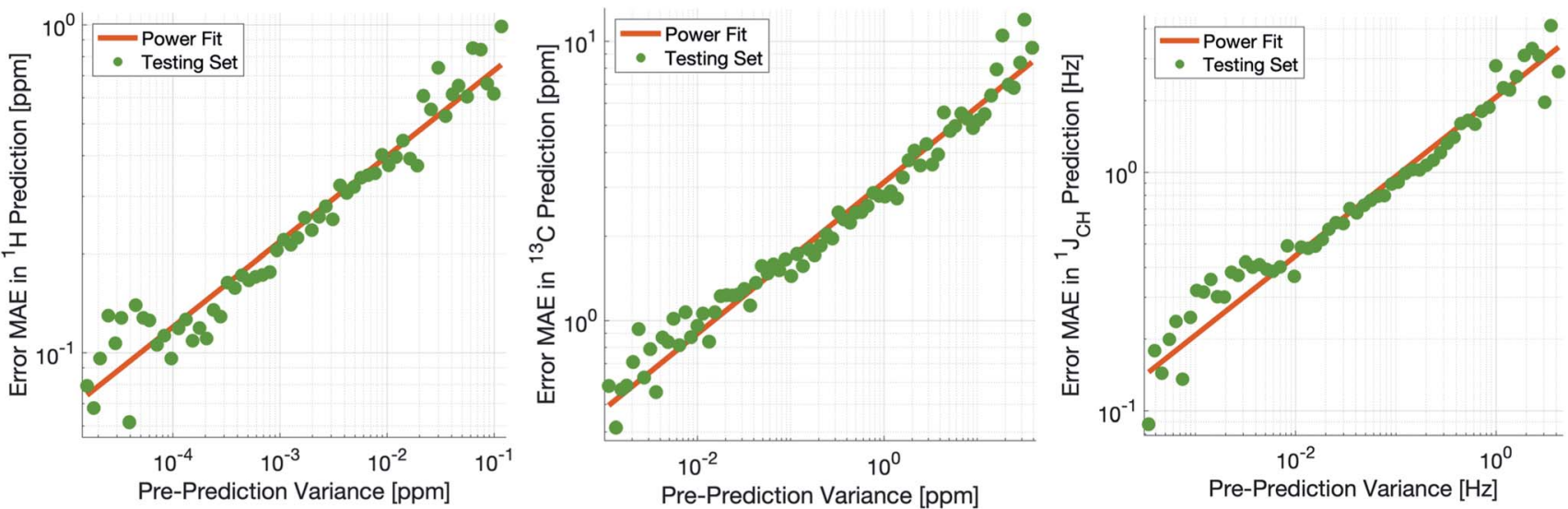

\begin{tabular}{|c|c|c|c|c|}
\hline Variance [ppm] & Envs & MAE [ppm] & RMSE [ppm] & MaxE [ppm] \\
\hline $\mathrm{V}>0.1$ & 5 & 3.53 & 5.30 & 11.22 \\
\hline $0.01<\mathrm{V}<0.1$ & 266 & 0.51 & 0.67 & 1.94 \\
\hline $0.001<\mathrm{V}<0.01$ & 3492 & 0.27 & 0.36 & 2.16 \\
\hline $\mathrm{V}<0.001$ & 3613 & 0.15 & 0.21 & 1.36 \\
\hline
\end{tabular}

\begin{tabular}{|c|c|c|c|c|}
\hline Variance [ppm] & Envs & MAE [ppm] & RMSE [ppm] & MaxE [ppm] \\
\hline $\mathrm{V}>10$ & 213 & 7.72 & 10.78 & 63.33 \\
\hline $1<\mathrm{V}<10$ & 1899 & 3.88 & 5.33 & 37.87 \\
\hline $0.1<\mathrm{V}<1$ & 2978 & 2.17 & 3.03 & 23.13 \\
\hline $\mathrm{V}<0.1$ & 2432 & 1.22 & 1.76 & 10.05 \\
\hline
\end{tabular}

\begin{tabular}{|c|c|c|c|c|}
\hline Variance $[\mathrm{Hz}]$ & Envs & MAE $[\mathrm{Hz}]$ & RMSE $[\mathrm{Hz}]$ & MaxE $[\mathrm{Hz}]$ \\
\hline $\mathrm{V}>10$ & 9 & 7.11 & 9.71 & 24.63 \\
\hline $1<\mathrm{V}<10$ & 198 & 2.97 & 4.37 & 24.40 \\
\hline $0.1<\mathrm{V}<1$ & 2586 & 1.21 & 1.65 & 13.37 \\
\hline $\mathrm{V}<0.1$ & 4995 & 0.60 & 0.82 & 4.91 \\
\hline
\end{tabular}

Fig. 3 (Top) Correlation between pre-prediction variance and prediction error between DFT and IMPRESSION for $\delta^{1} \mathrm{H}$ (left), $\delta^{13} \mathrm{C}$ (centre) and ${ }^{1} \mathrm{~J}_{\mathrm{CH}}$ couplings (right) on the test set. The prediction errors were binned by variance and an average error (MAE) was produced for each bin. (Bottom) Error metrics for different variance ranges.

system is indeed very sensitive to the 3-dimensional relationships of the atoms in the structure. However this same sensitivity also makes IMPRESSION less accurate for chemical environments which are not very similar to environments across the 882 molecular structures used to train IMPRESSION.

Crucially, we are able to a priori identify poorly described environments using the same variance-based approach used to generate the training set. By assessing the variance in the prediction of a given NMR parameter across a 5 -fold crossvalidation, we can quantify our confidence in each individual prediction since environments which are poorly described by the chemical structures in the training set will have high variance in this cross-validation. There is indeed a clear correlation of variance against prediction error for the independent test set (Fig. 3). The tables in Fig. 3 suggest that the bulk of the environments are predicted very accurately, and that the high variance environments are the dominant source of the large outliers.

In principle, removing IMPRESSION-predicted values which show high variances in cross-validation should provide a "preprediction variance filter" that will substantially improve the quality of, and thus the confidence in, IMPRESSION predictions. Selecting an appropriate variance cut-off for each NMR parameter is then simply a balance between desired prediction quality and the number of predictions which will be excluded by that cut-off. Reports of DFT accuracy with respect to experiment for ${ }^{1} \mathrm{H}$ and ${ }^{13} \mathrm{C}$ chemical shift predictions vary significantly, but typically in the range of $0.2-0.4 \mathrm{ppm} / 2-4 \mathrm{ppm}$, with the best reported accuracies down to $<0.2 /<2 \mathrm{ppm}$ (ref. 16 and 17) in optimal cases. Similarly, Buevich et al. recently highlighted that current best-in-class DFT methods predict ${ }^{1} J_{\mathrm{CH}}$ experimental values with accuracies of $2-4 \mathrm{~Hz}$, when presenting an optimised workflow for calculating ${ }^{1} J_{\mathrm{CH}}$ values which achieved an RMSE of $1.61 \mathrm{~Hz}$.
We therefore identified variance cut-offs for IMPRESSION predictions that provide a good compromise between accuracy and excluded values for the test set, which were found to be $1 \mathrm{~Hz}$ for ${ }^{1} J_{\mathrm{CH}}, 0.1 \mathrm{ppm}$ for $\delta^{1} \mathrm{H}$ and $5 \mathrm{ppm}$ for $\delta^{13} \mathrm{C}$. Applying these prevariance filter values improves the fits between IMPRESSION and DFT to levels that are comparable with literature reports for MAE/RMSE of DFT vs. experiment (MaxE is rarely reported for large experimental validations, but the reader can find comparators from our experimental validations described below in Section 2.3). For $\delta^{1} \mathrm{H}$ the $0.1 \mathrm{ppm}$ filter excludes 5 environments $(<0.1 \%)$ and improves the fit to $\mathrm{MAE}=0.23 \mathrm{ppm}$, RMSE $=0.32 \mathrm{ppm}$; MaxE $=2.16 \mathrm{ppm}$. For $\delta^{13} \mathrm{C}$ a $5 \mathrm{ppm}$ filter provided a good fit $(\mathrm{MAE}=2.17 \mathrm{ppm} ; \mathrm{RMSE}=3.25 \mathrm{ppm}$; MaxE $=37.87 \mathrm{ppm})$ while excluding $538(\sim 7.2 \%)$ of the environments. For ${ }^{1} J_{\mathrm{CH}}$ a $1 \mathrm{~Hz}$ filter improved the fit to $\mathrm{MAE}=0.81 \mathrm{~Hz}, \mathrm{RMSE}=$ $1.17 \mathrm{~Hz} ; \operatorname{MaxE}=13.37 \mathrm{~Hz}$ while discarding only $207(<3 \%)$ of the environments.

As highlighted by the learning curves, further improvement to the machine predictions of DFT NMR results can be made by increasing the size of the DFT-derived training dataset by around an order of magnitude. However at this stage variancefiltered IMPRESSION compares well enough with respect to DFT that it was taken forward. It should also be noted at this point that IMPRESSION only accelerates NMR prediction, it does not accelerate the 3D structure generation by DFT (which can still take hours/days). This overall time, i.e. 3D structure generation + NMR prediction, could be reduced further by using 3D structures derived from molecular mechanics rather than DFT. While not the key focus here, the use of molecular mechanics structures as inputs to a re-trained IMPRESSION machine was explored. While practical, this resulted in a $\sim 30-50 \%$ increase in the average prediction errors for $\delta^{1} \mathrm{H}$ and ${ }^{1} J_{\mathrm{CH}}$ presumably arising from a mismatch between the detail of molecular mechanics geometries and those used to calculate the DFT 
NMR parameters (see Section S2 in the ESI for details $\dagger$ ). Interestingly, $\delta^{13} \mathrm{C}$ predictions were relatively insensitive to this change, perhaps reflecting better description of carbon environments by molecular mechanics forcefields. This is an exciting avenue to explore further, but to focus the discussion here on the ability of IMPRESSION to reproduce DFT NMR predictions, the subsequent experimental comparisons are based on the IMPRESSION machine trained on the same DFTgeometries used for the DFT NMR predictions.

\subsection{Performance relative to experiment}

Naturally, a key test of IMPRESSION is its ability to reproduce DFT predictions of experimental values of relevant compounds. To test this for ${ }^{1} J_{\mathrm{CH}}$, a validation set of 608 experimental ${ }^{1} J_{\mathrm{CH}}$ values were taken from structures collated by Venkata et al. ${ }^{23}$ which contain $\mathrm{C}, \mathrm{H}, \mathrm{N}, \mathrm{O}$ and F elements only. Firstly, we checked the ability of our $\omega \mathrm{b} 97 \mathrm{xd} / 6-311 \mathrm{~g}(\mathrm{~d}, \mathrm{p})$ DFT method itself to reproduce these experimental results. It should be noted in the subsequent analysis that all DFT and IMPRESSION predictions were based on the single conformers that Venkata et al. reported for each compound. While not making the predictions entirely experimentally relevant, it allows direct comparison between DFT and IMPRESSION NMR predictions for this data. Calculating the 608 couplings with $\omega \mathrm{b} 97 \mathrm{xd} / 6-311 \mathrm{~g}(\mathrm{~d}, \mathrm{p})$ took 156 CPU hours and initially gave a relatively poor fit to experiment $(\mathrm{MAE}=10.92 \mathrm{~Hz})$ but with a systematic offset from the experimental data by an average of $-10.91 \mathrm{~Hz}$. Adding this systematic offset to the DFT-predicted values provided a good fit between DFT and experiment $(\mathrm{MAE}=2.16 \mathrm{~Hz}$; RMSE $=3.33 \mathrm{~Hz}$; $\mathrm{MaxE}=$ $20.05 \mathrm{~Hz}$ ) and this was used for all subsequent comparisons to experiment based on this DFT method. As IMPRESSION is trained on DFT data computed with this same wb97xd/6$311 \mathrm{~g}(\mathrm{~d}, \mathrm{p})$ method and both methods use only single conformer predictions for each molecule, then these statistics represent a practical limit for the accuracy that we might expect from IMPRESSION on this experimental data.

IMPRESSION took only 60 CPU seconds to predict the full set of $612{ }^{1} J_{\mathrm{CH}}$ values but with some substantial outliers (MAE = $4.52 \mathrm{~Hz}$; RMSE $=10.49 \mathrm{~Hz}$; MaxE $=120.3 \mathrm{~Hz}$ ). Applying the $1 \mathrm{~Hz}$ variance filter gave: $\mathrm{MAE}=2.01 \mathrm{~Hz}, \mathrm{RMSE}=2.69 \mathrm{~Hz}, \mathrm{MaxE}=$ $10.01 \mathrm{~Hz}$ (removing 143 values) which was essentially identical accuracy to that obtained from the DFT method for these same filtered environments: $\mathrm{MAE}=1.83 \mathrm{~Hz}, \mathrm{RMSD}=2.60 \mathrm{~Hz}$, MaxE $=14.63 \mathrm{~Hz}$. An overlay of the error distributions for DFT and the $1 \mathrm{~Hz}$ variance-filtered IMPRESSION vs. the experimental values (Fig. 4) demonstrates the comparability between machine learning and DFT for ${ }^{1} J_{\mathrm{CH}}$ predictions. This represents quite excellent performance of the machine for reproducing experimental data in just a few seconds, with quality for the majority of environments as good as the best MAEs (1.5-4 Hz) described by Buevich et al. as typical for DFT methods, with $<25 \%$ of the values being tagged as unreliable by the variance filter. Of course, if a slight loss in prediction quality is acceptable for a given study, then more predicted values could be retained by using a slightly looser variance-filter.

Similar accuracy could be obtained for IMPRESSION predictions of $734{ }^{1} \mathrm{H}$ chemical shifts for 36 structures reported by Smith and Goodman ${ }^{44}$ in their DP4 dataset (again, single conformers were used for both DFT and IMPRESSION predictions). IMPRESSION predictions gave MAE $=0.29 \mathrm{ppm}$, RMSD $=0.38 \mathrm{ppm}, \mathrm{MaxE}=1.59 \mathrm{ppm}$ with a variance filter of $0.1 \mathrm{ppm}$ but in this case no environments were removed with the variance filter and provided essentially the same outcomes as the wb97xd/6-311g(d,p) DFT method on the same single conformer structures $(\mathrm{MAE}=0.28 \mathrm{ppm}$, RMSE $0.37 \mathrm{ppm}$, MaxE $1.62 \mathrm{ppm}$, see Fig. 4 for an overlay of errors). The IMPRESSION predictions for $\delta^{13} \mathrm{C}$ using the $5 \mathrm{ppm}$ variance filter identified during training and testing of the machine compared slightly less well to the DP4 experimental dataset $(\mathrm{MAE}=3.44 \mathrm{ppm}, \mathrm{RMSE}=$ $4.30 \mathrm{ppm}, \mathrm{MaxE}=13.06 \mathrm{ppm}$, removing 24 environments) than DFT $(\mathrm{MAE}=2.78 \mathrm{ppm}, \mathrm{RMSE}=3.48 \mathrm{ppm}, \mathrm{MaxE}=14.33 \mathrm{ppm})$. A tighter $1 \mathrm{ppm}$ variance filter for the $\delta^{13} \mathrm{C}$ predictions was examined, but gave only a slight improvement in prediction quality $\mathrm{MAE}=3.20 \mathrm{ppm}, \mathrm{RMSE}=4.00 \mathrm{ppm}, \operatorname{MaxE}=13.03 \mathrm{ppm}$ while removing 120 out of the 458 carbon environments.

At every stage in this study we found that the IMPRESSION $\delta^{13} \mathrm{C}$ predictions have a wider distribution of errors than the other NMR parameters when compared to the quality of the
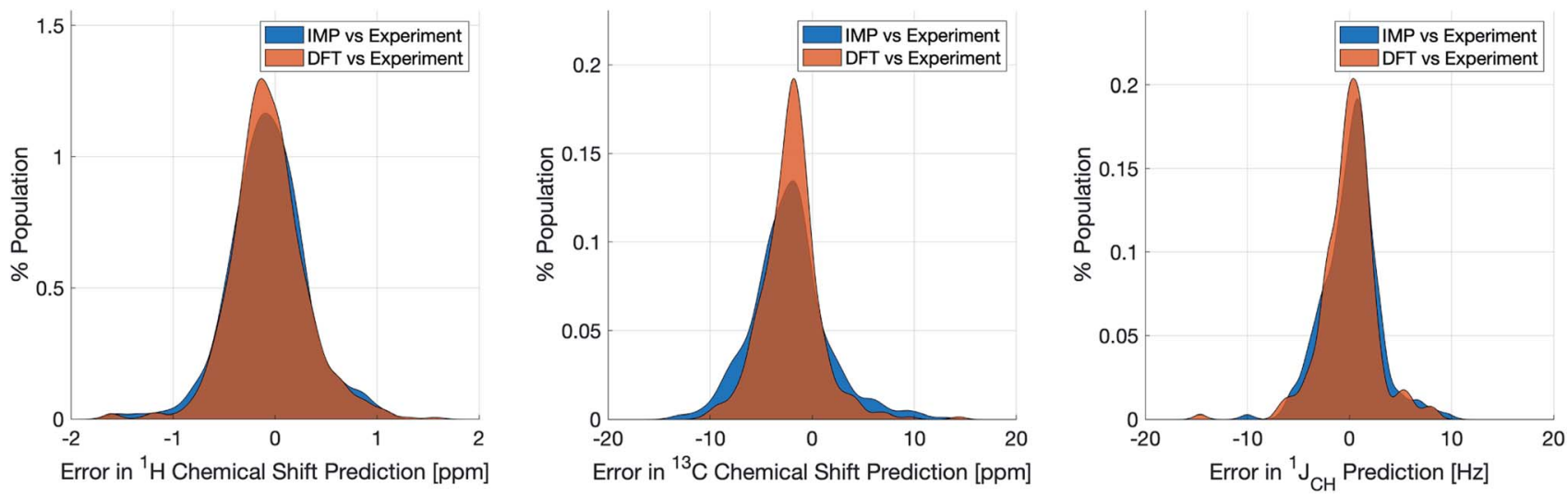

Fig. 4 Distribution of errors for machine learning NMR predictions and DFT calculations when compared to the relevant experimental validation dataset for $\delta^{1} \mathrm{H}$ (left), $\delta^{13} \mathrm{C}$ (centre) and ${ }^{1} \mathrm{~J}_{\mathrm{CH}}$ couplings (right). Variance filters applied to IMPRESSION predictions: $\delta^{1} \mathrm{H}=0.1 \mathrm{ppm}(0$ of 734 environments removed), $\delta^{13} \mathrm{C}=5 \mathrm{ppm}$ (24 of 457 environments removed), ${ }^{1} \mathrm{~J}_{\mathrm{CH}}=1 \mathrm{~Hz}$ (143 of 608 environments removed). 
DFT from which they are trained. This is unsurprising given that the structural environments of ${ }^{13} \mathrm{C}$ nuclei in molecules are inherently more complex than ${ }^{1} \mathrm{H}$ given the higher valency and thus more complex bonding environments and geometries, so in future development, larger training datasets focussed on optimising $\delta^{13} \mathrm{C}$ predictions will be beneficial.

\subsection{3-Dimensional structure discrimination}

A demanding test of IMPRESSION is in its ability to predict and discriminate experimental NMR data for stereoisomeric compounds i.e. those that differ only in their 3-dimensional structure, but not connectivity. Even though IMPRESSION has not been explicitly trained to deal with multiple conformers/ isomers of any one compound, 3-dimensional variation is implicit within the varied chemical structural space of the adaptively sampled training set. Buevich et al. recently demonstrated $^{5}$ that DFT prediction of ${ }^{1} J_{\mathrm{CH}}$ values can successfully discriminate the naturally occurring structure $\mathbf{1}$ of the polycyclic alkaloid strychnine (Fig. 5, centre) from 12 other diastereomers (see ESI Section S5 $†$ for the structures) based on comparison with the experimental ${ }^{1} J_{\mathrm{CH}}$ values of the natural product. Pleasingly, the same test conducted with IMPRESSION-predicted ${ }^{1} J_{\mathrm{CH}}$ values (blue bars in Fig. 5 , left) also correctly identifies the natural product diastereomer 1a as having the smallest error $(\mathrm{MAE}=1.87 \mathrm{~Hz}$; RMSE $=2.50 \mathrm{~Hz}$; MaxE $=6.19 \mathrm{~Hz}$ ). The error for the correct structure is $\sim 30 \%$ lower than the diastereomer with the second lowest error 6 $(\mathrm{MAE}=2.48 \mathrm{~Hz} ; \mathrm{RMSE}=3.38 \mathrm{~Hz} ; \operatorname{MaxE}=8.42 \mathrm{~Hz})$ and this is very similar to the discrimination offered by $\omega \mathrm{b} 97 \mathrm{xd} / 6-$ $311 \mathrm{~g}(\mathrm{~d}, \mathrm{p})$ (red bars in Fig. 5). Indeed IMPRESSION could also distinguish between the 3-dimensional structures of 1a, the lowest energy conformer of the natural product $(97 \%$ population in solution), and $\mathbf{1 b}$ which is the second lowest energy conformer (3\% population in solution) ${ }^{45}$ So while the absolute accuracy of IMPRESSION for predicting ${ }^{1} J_{\mathrm{CH}}$ values for strychnine ( $\mathrm{MAE}=1.87 \mathrm{~Hz}$ ) is slightly lower than that obtained from the DFT method (MAE $=1.31 \mathrm{~Hz})$, its discriminating power between structural isomers is nearly the same.

Combining IMPRESSION predictions for ${ }^{1} J_{\mathrm{CH}}$ with ${ }^{1} \mathrm{H}$ and ${ }^{13} \mathrm{C}$ chemical shifts also provides correct identification of the naturally occurring structure, but IMPRESSION and DFT now both see structure 2 as the next best candidate (Fig. 5, right). This is due to the experimental $\delta^{1} \mathrm{H}$ values having better agreement with the predictions for diastereomer 2 than $1 \mathrm{a}$ for DFT and also IMPRESSION. While this is obviously problematic for structure elucidation purposes, it clearly arises because of a deficiency in the DFT prediction of ${ }^{1} \mathrm{H}$ chemical shifts, which is then faithfully reproduced by IMPRESSION. For the individual MAE values across all three parameters see ESI Section S5.†

Similarly, we found that IMPRESSION predictions can be used to correctly assign the diastereotopic protons in strychnine. IMPRESSION and DFT predictions of ${ }^{1} J_{\mathrm{CH}}$ for the diastereotopic protons in strychnine were consistently in line with each other (details can be found in Section S4 of the ESI†े) and for the three methylene groups where there is a significant difference $(\gg 2 \mathrm{~Hz})$ in experimental ${ }^{1} J_{\mathrm{CH}}$ values both methods correctly assign these protons (Fig. S16†).

Finally, we validated IMPRESSION chemical shift predictions for natural product structures. We conducted DFT and IMPRESSION predictions on structures from a recent report which suggested structural reassignments for oxiranecontaining natural products on the basis of DU8+ DFT calculations ${ }^{46}$ To avoid complications with incorrect DFT prediction of conformer energies leading to poor population averaging of NMR parameters from the constituent conformers, we limited the validation to 'rigid' structures in the report that contained only one dominant conformer after conformational searching. Pleasingly, while our results did not always agree with the DU8+ analysis, IMPRESSION was just as effective as our underlying $\omega \mathrm{b} 97 \mathrm{xd} / 6-311 \mathrm{~g}(\mathrm{~d}, \mathrm{p})$ DFT method in discriminating each original and revised chemical structure (see Section S3 in the ESI for
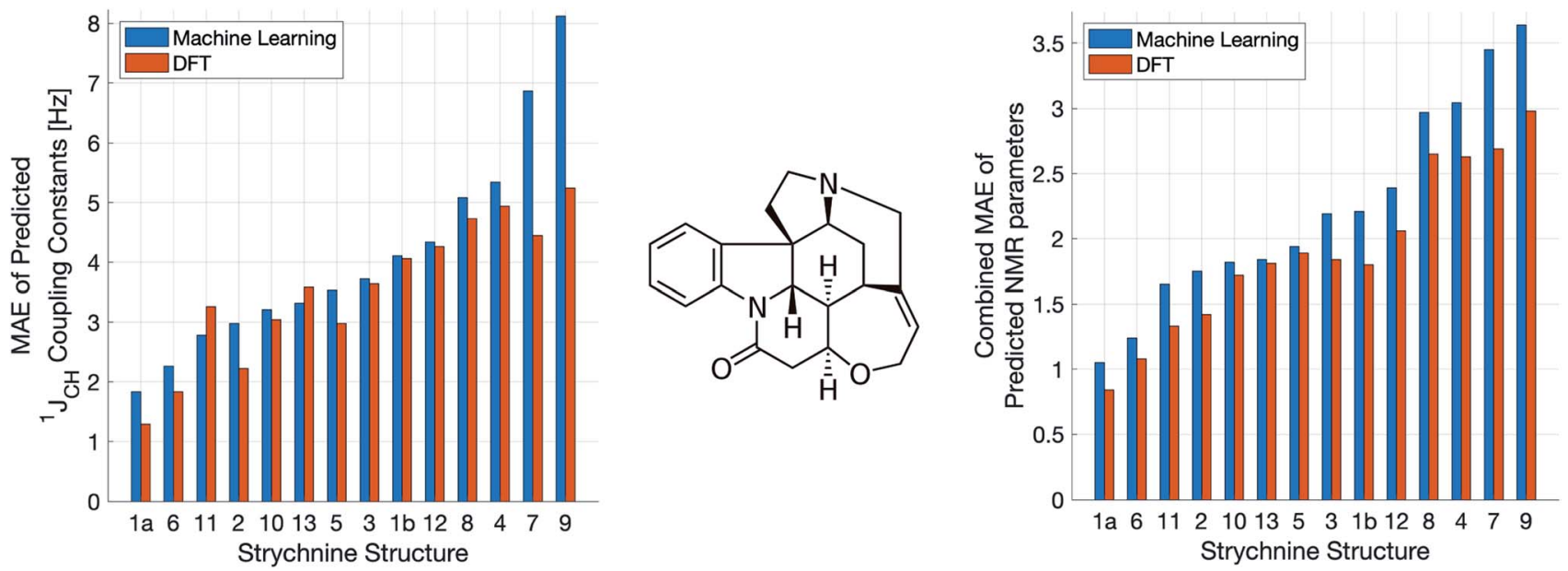

Fig. 5 Errors from comparison of NMR experimental data of the natural product strychnine (centre) to IMPRESSION (blue) and DFT (red) predictions for 13 diastereomers of strychnine, including two conformers for the natural product 1: the lowest energy 1a (>97\% populated) and the next lowest energy $1 \mathrm{~b}\left(<3 \%\right.$ populated). The left hand plot shows MAE for ${ }^{1} \mathrm{~J}_{\mathrm{CH}}$ while the right hand plot shows the geometric mean absolute error for all NMR parameters $\left(\delta^{1} \mathrm{H}, \delta^{13} \mathrm{C}\right.$ and $\left.{ }^{1} \mathrm{~J}_{\mathrm{CH}}\right)$ combined. Variance filters applied to predictions: $\delta^{1} \mathrm{H}=0.1 \mathrm{ppm}, \delta^{13} \mathrm{C}=5 \mathrm{ppm},{ }^{1} \mathrm{~J}_{\mathrm{CH}}=1 \mathrm{~Hz}$. 
more details $\dagger$ ). Once again this confirms that IMPRESSION is capable of making predictions that are of comparable quality to it's underlying DFT method $\omega \mathrm{b} 97 x d / 6-311 \mathrm{~g}(\mathrm{~d}, \mathrm{p})$, and thus any improvements in the DFT method used to train IMPRESSION will be subsequently expressed in the quality of IMPRESSION predictions.

\section{Conclusions}

In summary, this first generation IMPRESSION machine, trained on DFT-computed NMR parameters derived from a set of 3-dimensional structures is capable of reproducing DFTpredicted NMR parameters for a range of experimentally relevant systems with high accuracy but in a fraction of the time. Accurate and generalised prediction of NMR parameters for 3dimensional applications has not been addressed by previous machine learning systems but the confidence provided by the variance-filtered IMPRESSION results makes this tool essentially as robust for 3-dimensional applications to experimental systems as DFT. At this stage, the two primary sources of error in IMPRESSION predictions of experimental data are errors in the underlying DFT method on which it is trained (of which there can be several ${ }^{47-49}$ ) and the range of chemical space covered by the current IMPRESSION training set. We are working to improve both of these factors, as well as extending the predictions to multiple-bond scalar couplings for future generations of IMPRESSION, along with developing a more rigorous statistical treatment of the predicted values taking into account the pre-prediction variance.

\section{Conflicts of interest}

There are no conflicts to declare.

\section{Acknowledgements}

This work was carried out using the computational facilities of the Advanced Computing Research Centre, University of Bristol - http://www.bristol.ac.uk/acrc/. We thank Dr Peter Howe (Syngenta, UK) for useful discussions regarding the experimental ${ }^{1} J_{\mathrm{CH}}$ dataset used. WG thanks the EPSRC National Productivity Investment Fund (NPIF) for Doctoral Studentship funding. LAB thanks the Alan Turing Institute under the EPSRC grant EP/N510129/1. DRG acknowledges funding from the Royal Society as a University Research Fellow, and also from EPSRC grant EP/M022129/1. AJM thanks EPSRC for funding (EP/M022609/1, CCP-BioSim). LAB and DRG acknowledge support of this work through EPSRC grant EP/P021123/1. We further acknowledge the use of the following software: BayesianOptimization, ${ }^{50}$ Open Babel, ${ }^{51}$ Pybel, ${ }^{52}$ NumPy, ${ }^{53}$ OpenMP, ${ }^{54}$ F2PY. ${ }^{55}$

\section{Notes and references}

1 E. Pretsch, T. Clerc, J. Seibl and W. Simon, Tables of spectral data for structure determination of organic compounds, Springer Science \& Business Media, 2013.
2 W. Bremser, Anal. Chim. Acta, 1978, 103, 355-365.

3 S. Kuhn and S. R. Johnson, ACS Omega, 2019, 4, 7323-7329.

4 J. Aires-de Sousa, M. C. Hemmer and J. Gasteiger, Anal. Chem., 2002, 74, 80-90.

5 A. V. Buevich, J. Saurí, T. Parella, N. De Tommasi, G. Bifulco, R. T. Williamson and G. E. Martin, Chem. Commun., 2019, 55, 5781-5784.

$6 \mathrm{~J}$. Meiler, W. Maier, M. Will and R. Meusinger, J. Magn. Reson., 2002, 157, 242-252.

7 C. Haasnoot, F. A. de Leeuw and C. Altona, Tetrahedron, 1980, 36, 2783-2792.

8 B. Coxon, Adv. Carbohydr. Chem. Biochem., 2009, 62, 17-82.

9 A. Navarro-Vázquez, Magn. Reson. Chem., 2017, 55, 29-32.

10 M. W. Lodewyk, M. R. Siebert and D. J. Tantillo, Chem. Rev., 2011, 112, 1839-1862.

11 C. Steinmann, L. A. Bratholm, J. M. H. Olsen and J. Kongsted, J. Chem. Theory Comput., 2017, 13, 525-536.

12 A. S. Larsen, L. A. Bratholm, A. S. Christensen, M. Channir and J. H. Jensen, PeerJ, 2015, 3, e1344.

13 T. Helgaker, M. Jaszuński and M. Pecul, Prog. Nucl. Magn. Reson. Spectrosc., 2008, 4, 249-268.

14 S. N. Maximoff, J. E. Peralta, V. Barone and G. E. Scuseria, J. Chem. Theory Comput., 2005, 1, 541-545.

15 J. F. San, J. de la Vega García, R. Suardíaz, M. FernándezOliva, C. Pérez, R. Crespo-Otero and R. Contreras, Magn. Reson. Chem., 2013, 51, 775-787.

16 N. Grimblat, M. M. Zanardi and A. M. Sarotti, J. Org. Chem., 2015, 80, 12526-12534.

17 V. A. Semenov and L. B. Krivdin, Magn. Reson. Chem., 2019, DOI: $10.1002 / \mathrm{mrc} .4922$.

18 NMR Prediction Software from ACD/Labs, https:// www.acdlabs.com/products/adh/nmr/nmr_pred/.

19 NMR Prediction Software from Mestrelab, https:// mestrelab.com/software/mnova/nmr-predict/.

20 A. M. Castillo, A. Bernal, R. Dieden, L. Patiny and J. Wist, J. Cheminf., 2016, 8, 26.

21 A. J. Brandolini, NMRPredict, Modgraph Consultants Ltd, CA 92129, 2006.

22 F. M. Paruzzo, A. Hofstetter, F. Musil, S. De, M. Ceriotti and L. Emsley, Nat. Commun., 2018, 9, 4501.

23 C. Venkata, M. J. Forster, P. W. Howe and C. Steinbeck, PLoS One, 2014, 9, e111576.

24 M. Frisch, G. Trucks, H. Schlegel, G. Scuseria, M. Robb, J. Cheeseman, G. Scalmani, V. Barone, B. Mennucci, G. Petersson and others, Wallingford, CT, 2016, (For the full reference see the $\mathrm{ESI}_{\dagger}^{\dagger}$ ).

25 A. M. Teale, O. B. Lutnæs, T. Helgaker, D. J. Tozer and J. Gauss, J. Chem. Phys., 2013, 138, 024111.

26 C. Adamo and V. Barone, J. Chem. Phys., 1998, 108, 664-675. 27 A. McLean and G. Chandler, J. Chem. Phys., 1980, 72, 56395648.

28 R. Krishnan, J. S. Binkley, R. Seeger and J. A. Pople, J. Chem. Phys., 1980, 72, 650-654.

29 J.-D. Chai and M. Head-Gordon, J. Chem. Phys., 2008, 128, 084106.

30 W. Deng, J. R. Cheeseman and M. J. Frisch, J. Chem. Theory Comput., 2006, 2, 1028-1037. 
31 P. B. Wilson, M. Grootveld and S. C. L. Kamerlin, Magn. Reson. Chem., 2019, DOI: 10.1002/mrc.4940.

32 R. Laskowski, P. Blaha and F. Tran, CHESHIRE Chemical Shift Repository, 2019, accessed October 2nd, 2019.

33 H. S. Seung, M. Opper and H. Sompolinsky, Proc. 5th Ann. Work. Comp. Learn. Theory, New York, NY, USA, 1992, pp. 287-294.

34 M. Gastegger, J. Behler and P. Marquetand, Chem. Sci., 2017, 8, 6924-6935.

35 J. S. Smith, B. Nebgen, N. Lubbers, O. Isayev and A. E. Roitberg, J. Chem. Phys., 2018, 148, 241733.

36 C. R. Groom, I. J. Bruno, M. P. Lightfoot and S. C. Ward, Acta Crystallogr., Sect. B: Struct. Sci., Cryst. Eng. Mater., 2016, 72, 171-179.

37 C. Saunders, A. Gammerman and V. Vovk, Proceedings of the 15th International Conference on Machine Learning (ICML '98), 1998.

38 Y. Binev and J. Aires-de Sousa, J. Chem. Inf. Comput. Sci., 2004, 44, 940-945.

39 Y. Binev, M. M. Marques and J. Aires-de Sousa, J. Chem. Inf. Model., 2007, 47, 2089-2097.

40 M. Rupp, R. Ramakrishnan and O. A. Von Lilienfeld, J. Phys. Chem. Lett., 2015, 6, 3309-3313.

41 B. Huang and O. A. von Lilienfeld, arXiv preprint arXiv:1707.04146, 2017.

42 F. A. Faber, A. S. Christensen, B. Huang and O. A. von Lilienfeld, J. Chem. Phys., 2018, 148, 241717.
43 A. S. Christensen, L. A. Bratholm, S. Amabilino, J. C. Kromann, F. A. Faber, B. Huang, A. Tkatchenko, K. R. MÃijller and O. A. von Lilienfeld, QML: A Python Toolkit for Quantum Machine Learning, 2019, https:// github.com/qmlcode/qml.

44 S. G. Smith and J. M. Goodman, J. Am. Chem. Soc., 2010, 132, 12946-12959.

45 C. P. Butts, C. R. Jones and J. N. Harvey, Chem. Commun., 2011, 47, 1193-1195.

46 A. G. Kutateladze, D. M. Kuznetsov, A. A. Beloglazkina and T. Holt, J. Org. Chem., 2018, 83, 8341-8352.

47 M. A. Iron, J. Chem. Theory Comput., 2017, 13, 5798-5819.

48 A. Bagno, F. Rastrelli and G. Saielli, Chem.-Eur. J., 2006, 12, 5514-5525.

49 R. Laskowski, P. Blaha and F. Tran, Phys. Rev. B: Condens. Matter Mater. Phys., 2013, 87, 195130.

50 F. Nogueira, A Python implementation of global optimization with gaussian processes, 2019, https://github.com/fmfn/ BayesianOptimization.

51 N. M. O'Boyle, M. Banck, C. A. James, C. Morley, T. Vandermeersch and G. R. Hutchison, J. Cheminf., 2011, $3,33$.

52 N. M. O'Boyle, C. Morley and G. R. Hutchison, Chem. Cent. J., 2008, 2, 5.

53 T. E. Oliphant, A guide to NumPy, Trelgol Publishing USA, 2006, vol. 1.

54 L. Dagum and R. Menon, Comput. Sci. Eng., 1998, 46-55. 55 P. Peterson, Int. J. Comput. Sci. Eng., 2009, 4, 296-305. 